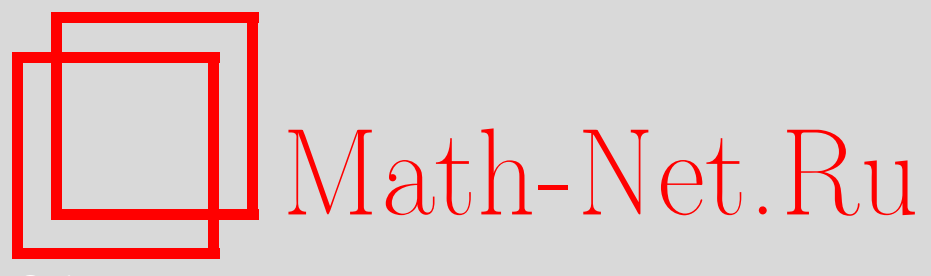

Дж.-Х. Ли, О. К. Пашаев, Солитонные решения резонансного нелинейного уравнения Шредингера с нетривиальными граничными условиями и билинейный метод Хироты, ТМФ, 2007, том 152, номер 1, 133-146

DOI: https://doi.org/10.4213/tmf6075

Использование Общероссийского математического портала Math-Net.Ru подразумевает, что вы прочитали и согласны с пользовательским соглашением http://www . mathnet.ru/rus/agreement

Параметры загрузки:

IP : 3.80 .253 .173

26 апреля 2023 г., 13:16:03

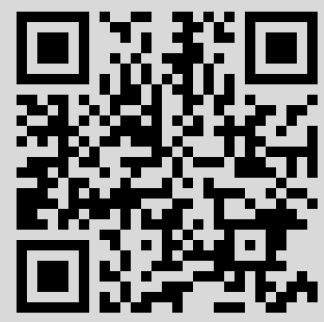




\section{СОЛИТОННЫЕ РЕШЕНИЯ РЕЗОНАНСНОГО НЕЛИНЕЙНОГО УРАВНЕНИЯ ШРЕДИНГЕРА С НЕТРИВИАЛЬНЫМИ ГРАНИЧНЫМИ УСЛОВИЯМИ И БИЛИНЕЙНЫЙ МЕТОД ХИРОТЫ}

В рамках билинейного метода Хироты рассмотрены физически значимые солитонные решения резонансного нелинейного уравнения Шредингера с нетривиальными граничными условиями, которое было недавно предложено для описания осесимметричных волн в холодной бесстолкновительной плазме. С помощью представления Маделунга модель преобразуется в являющуюся аналогом нелинейного уравнения Шредингера систему реакции-диффузии, для которой исследуются билинейное представление, солитонные решения и их взаимодействия.

Ключевые слова: резонансное нелинейное уравнение Шредингера, квантовый потенциал, холодная плазма, магнитоакустические волны, солитоны, метод Хироты.

\section{1. ВВЕДЕНИЕ}

Недавно для описания гравитации в низких размерностях (модель ДжакиваТейтельбойма) и отклика среды на действие квазимонохроматической волны с комплексной амплитудой $\psi(x, t)$, которая является медленно меняющейся функцией координат и времени, был предложен следующий новый интегрируемый вариант нелинейного уравнения Шредингера (НУШ) [1]:

$$
i \frac{\partial \psi}{\partial t}+\frac{\partial^{2} \psi}{\partial x^{2}}+\frac{\Lambda}{4}|\psi|^{2} \psi=s \frac{1}{|\psi|} \frac{\partial^{2}|\psi|}{\partial x^{2}} \psi
$$

Это уравнение было названо резонансным нелинейным уравнением Шредингера (РНУШ). Оно может рассматриваться как третий вариант НУШ, промежуточный между дефокусирующим и фокусирующим случаями. Несмотря на то что модель РНУШ является интегрируемой для любого значения параметра $s$, критическое значение $s=1$ разделяет две области значений этого параметра, в которых модель

*Institute of Mathematics, Academia Sinica, Taipei, 11529, Taiwan

${ }^{\dagger}$ Department of Mathematics, Izmir Institute of Technology, Urla-Izmir, 35430, Turkey. E-mail: oktaypashaev@iyte.edu.tr 
ведет себя по-разному. При $s<1$ модель сводится к обычному НУШ, однако при $s>1$ она к обычному НУШ не сводится, а сводится к системе реакции-диффузии (РД). В этом случае модели свойственны резонансные солитонные явления [1].

РНУШ может интерпретироваться, в частности, как уравнение, описывающее распространение солитонов НУШ в так называемом квантовом потеницале $U_{\mathrm{Q}}(x)=$ $|\psi|_{x x} /|\psi|$. Этот потенциал, отвечающий за возникновение квантового поведения, был введен де Бройлем [2] и использован впоследствии Бомом [3] при разработке теории скрытых параметров в квантовой механике. Он появляется также в стохастической механике [4]. Связи между такими неклассическими движениями с внутренним спиновым движением и шредингеровским дрожанием (zitterbewegung) pacсматривались в ряде работ (см. [5]). Квантовые потенциалы появляются также в предложенных нелинейных расширениях квантовой механики, связанных как со стохастическим квантованием [6], [7], так и с поправками, обусловленными квантовой гравитацией [8]. Было отмечено, что РНУШ, подобно обычному НУШ, может также быть выведено в рамках капиллярных моделей [9], [10].

В работе [11] было показано, что РНУШ появляется в задачах физики плазмы, где оно описывает распространение длинных одномерных магнитоакустических волн в холодной бесстолкновительной плазме, находящейся под действием поперечного магнитного поля. Удовлетворяющая РНУШ комплексная волновая функция представляет собой комбинацию плотности плазмы и потенциала скоростей, как в представлении Маделунга. Для построения решений, описывающих взаимодействие солитонных магнитоакустических волн, были предложены и использованы преобразования Беклунда-Дарбу вместе с новым ассоциированным нелинейным принципом суперпозиции. Такой подход требует рассмотрения решений РНУШ с нетривиальными граничными условиями на бесконечности. Цель настоящей работы построить такие решения с помощью билинейного метода Хироты и исследовать их взаимодействия.

\section{2. МАГНИТОАКУСТИЧЕСКИЕ ВОЛНЫ В ХОЛОДНОЙ ПЛАЗМЕ}

Уравнения динамики двухкомпонентной холодной бесстолкновительной плазмы во внешнем магнитном поле В [12], [13] для случая осесимметричного движения плазмы

$$
\mathbf{u}=u(x, t) \mathbf{e}_{x}, \quad \mathbf{B}=B(x, t) \mathbf{e}_{z}
$$

сводятся к виду [14]

$$
\begin{gathered}
\frac{\partial \rho}{\partial t}+\frac{\partial}{\partial x}(\rho u)=0, \\
\frac{\partial u}{\partial t}+u \frac{\partial u}{\partial x}+\frac{B}{\rho} \frac{\partial B}{\partial x}=0, \\
\frac{\partial}{\partial x}\left(\frac{1}{\rho} \frac{\partial B}{\partial x}\right)=B-\rho,
\end{gathered}
$$

где мы положили $B=1$ и плотность плазмы $\rho=1$ на бесконечности. Эта система, эквивалентная системе Уизема, была также получена Гуревичем и Мещеркиным [15]. Она описывает распространение нелинейных магнитоакустических волн 
в холодной плазме, находящейся в поперечном магнитном поле. Эль, Ходоровский и Тюрина показали [16], что система типа (3) появляется также при рассмотрении гиперзвукового потока, возникающего за телом тонкого профиля.

\section{3. ПРИБЛИЖЕНИЕ МЕЛКОЙ ВОДЫ}

Рассмотрим приближение мелкой воды для магнитоакустической системы (3). Изменяя масштаб пространственной и временной переменных следующим образом: $x^{\prime}=\beta x, t^{\prime}=\beta t$, имеем

$$
\begin{gathered}
\frac{\partial \rho}{\partial t^{\prime}}+\frac{\partial}{\partial x^{\prime}}(\rho u)=0 \\
\frac{\partial u}{\partial t^{\prime}}+u \frac{\partial u}{\partial x^{\prime}}+\frac{B}{\rho} \frac{\partial B}{\partial x^{\prime}}=0 \\
\beta^{2} \frac{\partial}{\partial x^{\prime}}\left(\frac{1}{\rho} \frac{\partial B}{\partial x^{\prime}}\right)=B-\rho .
\end{gathered}
$$

Разлагая $B$ в степенной ряд по параметру $\beta^{2}$ :

$$
B=\rho+\beta^{2} b_{2}\left(\rho, \rho_{x^{\prime}}, \rho_{x^{\prime} x^{\prime}}, \ldots\right)+O\left(\beta^{4}\right),
$$

и подставляя в уравнение (6), имеем

$$
b_{2}=\frac{\partial}{\partial x^{\prime}}\left(\frac{1}{\rho} \frac{\partial \rho}{\partial x^{\prime}}\right) .
$$

Подстановка разложения (7) в уравнение (5) дает

$$
\frac{\partial u}{\partial t^{\prime}}+u \frac{\partial u}{\partial x^{\prime}}+\frac{\partial \rho}{\partial x^{\prime}}+\beta^{2}\left[\frac{1}{\rho} \frac{\partial^{3} \rho}{\partial x^{\prime 3}}-\frac{2}{\rho^{2}} \frac{\partial \rho}{\partial x^{\prime}} \frac{\partial^{2} \rho}{\partial x^{\prime 2}}+\left(\frac{1}{\rho} \frac{\partial \rho}{\partial x^{\prime}}\right)^{3}\right]=0
$$

с точностью до членов порядка $O\left(\beta^{2}\right)$ включительно. В результате имеем следующую систему:

$$
\begin{gathered}
\frac{\partial \rho}{\partial t^{\prime}}+\frac{\partial}{\partial x^{\prime}}(\rho u)=0 \\
\frac{\partial u}{\partial t^{\prime}}+u \frac{\partial u}{\partial x^{\prime}}+\frac{\partial \rho}{\partial x^{\prime}}+\beta^{2} \frac{\partial}{\partial x^{\prime}}\left[\frac{1}{\rho} \frac{\partial^{2} \rho}{\partial x^{\prime 2}}-\frac{1}{2}\left(\frac{1}{\rho} \frac{\partial \rho}{\partial x^{\prime}}\right)^{2}\right]=0
\end{gathered}
$$

Данная система описывает распространение длинных магнитоакустических волн в холодной плазме плотности $\rho$, двигающихся со скоростью $u$ поперек магнитного поля, заданного формулами (2), (7) (см. [17], [18]). Дисперсия в этой системе отрицательна, т.е. волновая скорость уменьшается с увеличением волнового числа $k$.

\section{4. РЕЗОНАНСНОЕ НЕЛИНЕЙНОЕ УРАВНЕНИЕ ШРЕДИНГЕРА}

Вводя потенциал скоростей

$$
S(x, t)=-\frac{1}{2} \int^{x} u\left(x^{\prime}, t\right) d x^{\prime},
$$


так что $u=-2 \partial S / \partial x$, и интегрируя один раз уравнение $(11)$, получаем систему

$$
\begin{gathered}
\frac{\partial \rho}{\partial t}-2 \frac{\partial}{\partial x}\left(\rho \frac{\partial S}{\partial x}\right)=0 \\
-\frac{\partial S}{\partial t}+\left(\frac{\partial S}{\partial x}\right)^{2}+\frac{1}{2} \rho+\frac{\beta^{2}}{2}\left[\frac{1}{\rho} \frac{\partial^{2} \rho}{\partial x^{2}}-\frac{1}{2}\left(\frac{1}{\rho} \frac{\partial \rho}{\partial x}\right)^{2}\right]=0
\end{gathered}
$$

(штрихи опущены). Объединяя величины $\rho$ и $S$ в одну комплексную функцию

$$
\psi=\sqrt{\rho} e^{-i S}
$$

систему (12), (13) можно представить как НУШ с квантовым потенциалом,

$$
i \frac{\partial \psi}{\partial t}+\frac{\partial^{2} \psi}{\partial x^{2}}-\frac{1}{2}|\psi|^{2} \psi=\left(1+\beta^{2}\right) \frac{1}{|\psi|} \frac{\partial^{2}|\psi|}{\partial x^{2}} \psi
$$

т.е. как РНУШ (1), где $\Lambda=-2, s=1+\beta^{2}$ и использовано следующее выражение для квантового потенциала:

$$
\frac{1}{\rho} \frac{\partial^{2} \rho}{\partial x^{2}}-\frac{1}{2}\left(\frac{1}{\rho} \frac{\partial \rho}{\partial x}\right)^{2}=2 \frac{1}{\sqrt{\rho}} \frac{\partial^{2} \sqrt{\rho}}{\partial x^{2}} .
$$

Поскольку параметр $s>1$, уравнение (15) не может быть преобразовано в НУШ [1]. Однако посредством объединения величин $\rho$ и $S$ в пару вещественных функций

$$
e^{(+)}=\sqrt{\rho} e^{S / \beta}, \quad e^{(-)}=-\sqrt{\rho} e^{-S / \beta},
$$

так что $e^{(+)}>0, e^{(-)}<0$, уравнения $(12),(13)$ могут быть записаны в виде системы РД

$$
\mp \frac{\partial e^{( \pm)}}{\partial \tau}+\frac{\partial^{2} e^{( \pm)}}{\partial x^{\prime 2}}-\frac{1}{2 \beta^{2}} e^{(+)} e^{(-)} e^{( \pm)}=0,
$$

где $\tau=\beta t^{\prime}$.

Линеаризуя уравнение (15) вблизи решения конденсатного типа $\psi=\sqrt{\rho_{0}} e^{-i \rho_{0} t / 2}$, получаем дисперсию $\omega=\sqrt{\rho_{0}} k \sqrt{1-\beta^{2} k^{2} / \rho_{0}}$. Эта дисперсия отрицательна, т.е. волновая скорость уменьшается с увеличением волнового числа $k$, и ведет к неустойчивости коротких волн с волновым числом $k>k_{\mathrm{cr}}$, где $k_{\mathrm{cr}}=\sqrt{\rho_{0}} / \beta$. Однако такая неустойчивость является результатом усечения дисперсионного соотношения

$$
\omega^{2}=\rho_{0} \frac{k^{2}}{1+\beta^{2} k^{2} / \rho_{0}}
$$

для системы (4)-(6) и не соответствует никакому реальному физическому эффекту для волн на мелкой воде [19]. Хотя эта система линейно устойчива для всех значений волнового числа $k$, неизвестно, является ли она интегрируемой. Поэтому она не так подходит для изучения взаимодействий, как РНУШ, которое является вполне интегрируемым и обладает богатой совокупностью точных решений. 


\section{5. СТАЦИОНАРНЫЙ ПОТОК И СОЛИТОНЫ}

Рассмотрим теперь систему (10), (11) для стационарного потока. Она описывает движение с фиксированной скоростью $u(x, t)=u_{0}=$ const, для которой из уравнения непрерывности (10) следует, что $\partial \rho / \partial t+u_{0} \partial \rho / \partial x=0$ или что плотность жидкости имеет вид бегущей волны $\rho=\rho\left(x-u_{0} t\right)$, где $x^{\prime}=\beta x, t^{\prime}=\beta t$. Уравнение (11) в этом случае дает

$$
\rho+\left[\frac{1}{\rho} \frac{\partial^{2} \rho}{\partial x^{2}}-\frac{1}{2}\left(\frac{1}{\rho} \frac{\partial \rho}{\partial x}\right)^{2}\right]=a=\text { const } .
$$

Последнее уравнение имеет простую физическую интерпретацию. Движение с постоянной скоростью означает, что сумма всех сил, действующих на систему, равна нулю. В нашем случае уравнение (20) описывает компенсацию нелинейности за счет квантового потенциала. С учетом (16) это уравнение дает

$$
\rho+2 \frac{1}{\sqrt{\rho}} \frac{\partial^{2} \sqrt{\rho}}{\partial x^{2}}=a .
$$

Тогда в переменных $y(z)=\sqrt{\rho}, z=x-u_{0} t$ имеем нелинейное уравнение

$$
\frac{d^{2} y}{d z^{2}}-\frac{a}{2} y+\frac{1}{2} y^{3}=0
$$

или, умножая на $y^{\prime}$ и один раз интегрируя,

$$
\left(\frac{d y}{d z}\right)^{2}-\frac{a}{2} y^{2}+\frac{1}{4} y^{4}=2 b=\text { const } .
$$

Это уравнение имеет решение

$$
y=2 p \operatorname{dn}\left[p\left(x-u_{0} t\right), \kappa\right]
$$

где $\mathrm{dn}$ - эллиптическая функция Якоби с модулем $\kappa$, а $p$ - произвольная константа, которая связана с константами интегрирования $a>0$ и $b<0$ соотношениями

$$
\frac{a}{2 p^{2}}=1+\kappa^{\prime 2}, \quad \frac{b}{2 p^{4}}=-\kappa^{\prime 2}
$$

в которых $\kappa^{\prime}=\sqrt{1-\kappa^{2}}$ - дополнительный модуль эллиптической функции Якоби. Эти соотношения определяют связь между константами интегрирования

$$
a=2 p^{2}\left(1-\frac{b}{2 p^{4}}\right)
$$

и модуль $\kappa=\sqrt{1+b /\left(2 p^{4}\right)}$. Тогда для плотности $\rho$ имеем решение вида бегущей волны

$$
\rho(x, t)=4 p^{2} \operatorname{dn}^{2}\left[p\left(x-u_{0} t\right), \kappa\right]
$$


При фиксированном потенциале $(21)$ из уравнения (13) для $u_{0}=-(2 / \beta) \partial S / \partial x$ имеем уравнение Гамильтона-Якоби

$$
-\frac{1}{\beta} \frac{\partial S}{\partial t}+\frac{1}{\beta^{2}}\left(\frac{\partial S}{\partial x}\right)^{2}+\frac{a}{2}=0
$$

которое имеет решение вида

$$
S=\beta\left[S_{0}-\frac{u_{0}}{2} x+\left(\frac{u_{0}^{2}}{4}+\frac{a}{2}\right) t\right],
$$

или, с учетом (26),

$$
S=\beta\left[S_{0}-\frac{u_{0}}{2} x+\left(\frac{u_{0}^{2}}{4}+p^{2}\left(2-\kappa^{2}\right)\right) t\right] .
$$

В вырожденном случае $\kappa=1$, который отвечает случаю $b=0$ и, следовательно, $a=2 p^{2}$, эллиптическое решение $(27),(30)$ превращается в солитон

$$
\begin{gathered}
\rho(x, t)=4 p^{2} \operatorname{sch}^{2}\left[p\left(x-u_{0} t\right)\right], \\
S=\beta\left[S_{0}-\frac{u_{0}}{2} x+\left(\frac{u_{0}^{2}}{4}+p^{2}\right) t\right] .
\end{gathered}
$$

Бегущая волна более общего вида возникает при рассмотрении решения системы $(10),(11)$ вида $u(x, t)=u\left(x-u_{0} t\right), \rho(x, t)=\rho\left(x-u_{0} t\right)$. В таком случае уравнение $(10)$ влечет $d\left[\left(u-u_{0}\right) \rho\right] / d z=0$, где $z=x-u_{0} t$, и

$$
u=u_{0}+\frac{C}{\rho}
$$

где $C=$ const. Подставляя (32) в уравнение (11) и один раз интегрируя, имеем

$$
\frac{1}{2} \frac{C^{2}}{\rho^{2}}+\rho+\left[\frac{1}{\rho} \frac{d^{2} \rho}{d z^{2}}-\frac{1}{2}\left(\frac{1}{\rho} \frac{d \rho}{d z}\right)^{2}\right]=A=\text { const } .
$$

Умножая на $\rho^{2}$ и один раз дифференцируя, получаем уравнение Кортевега-де Фриза вида бегущей волны

$$
\rho\left(\frac{d^{3} \rho}{d z^{3}}+3 \rho \frac{d \rho}{d z}-2 A \frac{d \rho}{d z}\right)=0,
$$

из которого однократным интегрированием получаем

$$
\frac{d^{2} \rho}{d z^{2}}+\frac{3}{2} \rho^{2}-2 A \rho=B=\text { const }
$$

Это дает уравнение

$$
\left(\frac{d \rho}{d z}\right)^{2}=-\rho^{3}+2 A \rho^{2}+2 B \rho+C^{2}
$$

и решение

$$
\rho\left(x-u_{0} t\right)=\alpha_{1}+\left(\alpha_{3}-\alpha_{1}\right) \operatorname{dn}^{2}\left[\frac{1}{2}\left(\alpha_{3}-\alpha_{1}\right)^{1 / 2}\left(x-u_{0} t\right), \kappa\right]
$$


с модулем эллиптической функции $\kappa^{2}=\left(\alpha_{3}-\alpha_{2}\right) /\left(\alpha_{3}-\alpha_{1}\right)$, постоянными $\alpha_{1}, \alpha_{2}$, $\alpha_{3}$ и

$$
u\left(x-u_{0} t\right)=u_{0}-\frac{\left(\alpha_{1} \alpha_{2} \alpha_{3}\right)^{1 / 2}}{\rho} .
$$

Это решение впервые было получено в работе [18]. В частных случаях имеем следующие редукции.

1. Если $\alpha_{1}=\alpha_{2}=0$ и, соответственно, $\kappa^{2}=1$, то решение (37) сводится к решению (27) при выборе параметров $\alpha_{3}=4 k^{2}, b=-\alpha_{2} k^{2} / 2$ и скорости $u=u_{0}$.

2. Если $\alpha_{1}=\alpha_{2} \neq 0$, то снова $\kappa^{2}=1$, однако решение

$$
\rho\left(x-u_{0} t\right)=\alpha_{1}+\left(\alpha_{3}-\alpha_{1}\right) \operatorname{sch}^{2}\left[\frac{1}{2}\left(\alpha_{3}-\alpha_{1}\right)^{1 / 2}\left(x-u_{0} t\right)\right]
$$

имеет нетривиальные асимптотики. Актуальным для физики плазмы значением является $\alpha_{1}=1$, что дает $\lim _{|x| \rightarrow \infty} \rho=1$. Вводя обозначение $\alpha_{3} \equiv \sigma^{2}$, можно записать это решение в виде

$$
\rho\left(x-u_{0} t\right)=1+\frac{\left(\sigma^{2}-1\right)}{\operatorname{ch}^{2}\left[\frac{\sqrt{\sigma^{2}-1}}{2}\left(x-u_{0} t\right)\right]} .
$$

Используя полученные результаты, теперь можно построить решения РНУШ (15). Подставляя (27) и (30) в (14) и меняя параметр $k \equiv p / \beta$, имеем квазипериодическое решение

$$
\psi\left(x^{\prime}, t^{\prime}\right)=2 \beta k \operatorname{dn}\left[k\left(x^{\prime}-u_{0} t^{\prime}\right), \kappa\right] \exp \left\{-i\left[\phi_{0}-\frac{u_{0}}{2} x^{\prime}+\left[\frac{u_{0}^{2}}{4}+\beta^{2} k^{2}\left(2-\kappa^{2}\right)\right] t^{\prime}\right]\right\},
$$

где $x^{\prime}=\beta x, t^{\prime}=\beta t . \mathrm{B}$ пределе $\kappa=1$ оно дает солитон огибающей

$$
\psi\left(x^{\prime}, t^{\prime}\right)=2 \beta k \frac{1}{\operatorname{ch} k\left(x^{\prime}-u_{0} t^{\prime}\right)} \exp \left\{-i\left[\phi_{0}-\frac{u_{0}}{2} x^{\prime}+\left[\frac{u_{0}^{2}}{4}+\beta^{2} k^{2}\right] t^{\prime}\right]\right\} .
$$

Для системы РД (18) имеем, соответственно, диссипативное периодическое решение

$$
e^{( \pm)}\left(x^{\prime}, \tau\right)= \pm 2 \beta k \operatorname{dn}\left[k\left(x^{\prime}-v \tau\right), \kappa\right] \exp \left\{ \pm\left[\phi_{0}-\frac{v}{2} x^{\prime}+\left[\frac{v^{2}}{4}+k^{2}\left(2-\kappa^{2}\right)\right] \tau\right]\right\}
$$

где скорость $v \equiv u_{0} / \beta, k \equiv p / \beta$, и диссипативный аналог солитона огибающей

$$
e^{( \pm)}\left(x^{\prime}, \tau\right)= \pm 2 \beta k \frac{1}{\operatorname{ch} k\left(x^{\prime}-v \tau\right)} \exp \left\{ \pm\left[\phi_{0}-\frac{v}{2} x^{\prime}+\left[\frac{v^{2}}{4}+k^{2}\right] \tau\right]\right\}
$$

- так называемое диссипатонное решение [1].

\section{6. БИЛИНЕЙНАЯ ФОРМА И СОЛИТОНЫ}

6.1. Тривиальные граничные условия. Представление РНУШ (15) в виде системы РД (18) является ключевым моментом в построении многосолитонных решений. Благодаря алгебраическому сходству системы РД с НУШ нетрудно написать 
билинейное представление для системы РД. Выражая две вещественные функции $e^{(+)}, e^{(-)}$через три вещественные функции:

$$
e^{( \pm)}=2 \beta \frac{G^{ \pm}}{F}
$$

имеем следующую билинейную систему уравнений:

$$
\left( \pm D_{\tau}-D_{x}^{2}\right)\left(G^{( \pm)} \cdot F\right)=0, \quad D_{x}^{2}(F \cdot F)=-2 G^{(+)} G^{(-)} .
$$

Соответствующее решение РНУШ (15) имеет вид

$$
|\psi(x, t)|^{2}=\rho=-e^{(+)} e^{(-)}=2 \beta^{2} \frac{D_{x}^{2}(F \cdot F)}{F^{2}}=4 \beta^{2} \frac{\partial^{2} \ln F}{\partial x^{2}} .
$$

Один диссипатон дается следующим решением системы (46): $G^{ \pm}= \pm e^{\eta_{1}^{ \pm}}, F=$ $1+e^{\eta_{1}^{+}+\eta_{1}^{-}+\phi_{1,1}}, e^{\phi_{1,1}}=\left(k_{1}^{+}+k_{1}^{-}\right)^{-2}$, где $\eta_{1}^{ \pm} \equiv k_{1}^{ \pm} x \pm\left(k_{1}^{ \pm}\right)^{2} \tau+\eta_{1}^{ \pm(0)}$ и $k_{1}^{ \pm}, \eta_{1}^{ \pm(0)}-$ константы. Будучи выраженным через переопределенные параметры $k \equiv\left(k_{1}^{+}+k_{1}^{-}\right) / 2$, $v \equiv-\left(k_{1}^{+}-k_{1}^{-}\right)$, это решение приобретает вид (44). В пространстве параметров $(v, k)$ существует критическое значение $v_{\text {crit }}=2 k$ такое, что при $v<v_{\text {crit }}$ мы имеем $e^{ \pm} \rightarrow 0$ на бесконечностях, т.е. нулевые граничные условия для диссипатона. При критическом значении скорости в движущейся системе отсчета решение представляет собой стационарное состояние типа кинка $e^{ \pm}= \pm k e^{ \pm k \xi_{0}}(1 \mp$ th $k \xi)$ с постоянными асимптотиками $e^{ \pm} \rightarrow \pm 2 k e^{ \pm k \xi_{0}}$ при $x \rightarrow \mp \infty$ и $e^{ \pm} \rightarrow \pm 0$ при $x \rightarrow \pm \infty$. В надкритическом случае $v>v_{\text {crit }}$, имеем $e^{ \pm} \rightarrow \pm \infty$ при $x \rightarrow \mp \infty$ и $e^{ \pm} \rightarrow \pm 0$ при $x \rightarrow \pm \infty$.

Для двухдиссипатонного решения

$$
\begin{aligned}
G^{ \pm}= \pm & {\left[e^{\eta_{1}^{ \pm}}+e^{\eta_{2}^{ \pm}}+\left(\frac{\breve{k}_{12}^{ \pm \pm}}{k_{21}^{ \pm \mp} k_{11}^{+-}}\right)^{2} e^{\eta_{1}^{+}+\eta_{1}^{-}+\eta_{2}^{ \pm}}+\left(\frac{\breve{k}_{12}^{ \pm \pm}}{k_{12}^{ \pm \mp} k_{22}^{+-}}\right)^{2} e^{\eta_{2}^{+}+\eta_{2}^{-}+\eta_{1}^{ \pm}}\right] } \\
F=1 & +\frac{e^{\eta_{1}^{+}+\eta_{1}^{-}}}{\left(k_{11}^{+-}\right)^{2}}+\frac{e^{\eta_{1}^{+}+\eta_{2}^{-}}}{\left(k_{12}^{+-}\right)^{2}}+\frac{e^{\eta_{2}^{+}+\eta_{1}^{-}}}{\left(k_{21}^{+-}\right)^{2}}+\frac{e^{\eta_{2}^{+}+\eta_{2}^{-}}}{\left(k_{22}^{+-}\right)^{2}}+ \\
& +\left(\frac{\breve{k}_{12}^{++} \breve{k}_{12}^{--}}{k_{12}^{+-} k_{21}^{+-} k_{11}^{+-} k_{22}^{+-}}\right)^{2} e^{\eta_{1}^{+}+\eta_{1}^{-}+\eta_{2}^{+}+\eta_{2}^{-}}
\end{aligned}
$$

где $k_{i j}^{a b} \equiv k_{i}^{a}+k_{j}^{b}, \breve{k}_{i j}^{a b} \equiv k_{i}^{a}-k_{j}^{b}, \eta_{i}^{ \pm} \equiv k_{i}^{ \pm} x \pm\left(k_{i}^{ \pm}\right)^{2} \tau+\eta^{ \pm(0)}$. Это решение демонстрирует резонансный характер диссипатонных взаимодействий [1].

6.2. Нетривиальные граничные условия. Как было впервые замечено Хиротой, билинейная форма уравнений для НУШ дефокусирующего типа с ненулевыми граничными условиями должна быть изменена [20]. После подстановки представления (45) в систему (18) последняя расцепляется на систему уравнений

$$
\left( \pm D_{\tau}-D_{x}^{2}+\lambda\right)\left(G^{ \pm} \cdot F\right)=0, \quad\left(D_{x}^{2}-\lambda\right)(F \cdot F)=-2 G^{+} G^{-},
$$

где введена константа $\lambda$, подлежащая определению. Из соотношения (45) и второго уравнения (50) следует, что $-e^{(+)} e^{(-)}=-4 \beta^{2}\left[\lambda / 2-(\ln F)_{x x}\right]$. Разлагая величины $G^{ \pm}$и $F$ в степенной ряд Хироты:

$$
G^{ \pm}= \pm g_{0}^{ \pm}\left(1+\epsilon g_{1}^{ \pm}+\epsilon^{2} g_{2}^{ \pm}+\cdots\right), \quad F=1+\epsilon f_{1}+\epsilon^{2} f_{2}+\cdots
$$


и требуя выполнения условия $\lim _{|x| \rightarrow \infty}(\ln F)_{x x}=0$, получаем граничное условие

$$
\alpha_{1}=\lim _{|x| \rightarrow \infty}\left[-e^{(+)} e^{(-)}\right]=\lim _{|x| \rightarrow \infty}\left\{-4 \beta^{2}\left[\frac{\lambda}{2}-(\ln F)_{x x}\right]\right\}=-2 \beta^{2} \lambda
$$

которое определяет константу $\lambda=-\alpha_{1} /\left(2 \beta^{2}\right)$. В приближении нулевого порядка имеем систему

$$
\left( \pm D_{\tau}-D_{x}^{2}+\lambda\right)\left(g_{0}^{ \pm} \cdot 1\right)=0, \quad\left(D_{x}^{2}-\lambda\right)(1 \cdot 1)=2 g_{0}^{+} g_{0}^{-} .
$$

Эта система имеет решение вида $g_{0}^{ \pm}=\beta^{ \pm} e^{\theta^{ \pm}}$, где $\beta_{0}^{2} \equiv \beta^{+} \beta^{-}=-\lambda / 2=\alpha_{1} /\left(4 \beta^{2}\right)$, $\theta^{ \pm}= \pm k x \pm\left(k^{2}-\lambda\right) t, \beta^{ \pm}=\beta_{0} e^{ \pm \gamma_{0}}$. С учетом свойств производных Хироты

$$
\begin{aligned}
& D_{x}(f g \cdot h)=\frac{\partial f}{\partial x} g h+f D_{x}(g \cdot h) \\
& D_{x}^{2}(f g \cdot h)=\frac{\partial^{2} f}{\partial x^{2}} g h+2 \frac{\partial f}{\partial x} D_{x}(g \cdot h)+f D_{x}^{2}(g \cdot h)
\end{aligned}
$$

билинейная система принимает вид

$$
\begin{aligned}
& \left(\mp D_{\tau} \pm 2 k D_{x}+D_{x}^{2}\right)\left(\left(1+\epsilon g_{1}^{ \pm}+\epsilon^{2} g_{2}^{ \pm}+\cdots\right) \cdot\left(1+\epsilon f_{1}+\epsilon^{2} f_{2}+\cdots\right)\right)=0, \\
& \left(D_{x}^{2}+2 \beta_{0}^{2}\right)\left(\left(1+\epsilon f_{1}+\cdots\right) \cdot\left(1+\epsilon f_{1}+\cdots\right)\right)= \\
& \quad=2 \beta_{0}^{2}\left(1+\epsilon g_{1}^{+}+\cdots\right)\left(1+\epsilon g_{1}^{-}+\cdots\right) .
\end{aligned}
$$

6.2.1. Односолитонное решение. В первом порядке имеем систему

$$
\begin{gathered}
\left(\mp \partial_{\tau} \pm 2 k \partial_{x}+\partial_{x}^{2}\right) g_{1}^{ \pm}+\left( \pm \partial_{\tau} \mp 2 k \partial_{x}+\partial_{x}^{2}\right) f_{1}=0, \\
\left(\partial_{x}^{2}+2 \beta_{0}^{2}\right) f_{1}=\beta_{0}^{2}\left(g_{1}^{+}+g_{1}^{-}\right) .
\end{gathered}
$$

Рассматривая решение в виде $g_{1}^{ \pm}=a_{1}^{ \pm} e^{\eta_{1}}, f_{1}=b_{1} e^{\eta_{1}}$, где $\eta_{1}=k_{1} x+\omega_{1} \tau+\eta_{1}^{0}$, получаем $a_{1}^{+}=\gamma_{1} b_{1}, a_{1}^{-}=b_{1} / \gamma_{1}, \gamma_{1}=\left(\omega_{1}-2 k k_{1}+k_{1}^{2}\right) /\left(\omega_{1}-2 k k_{1}-k_{1}^{2}\right)$, где дисперсионная формула имеет вид

$$
\omega_{1}^{ \pm}=k_{1}\left(2 k \pm \sqrt{k_{1}^{2}+4 \beta_{0}^{2}}\right) .
$$

Стоит отметить, что, в отличие от случая решения для дефокусирующего НУШ, в нашем случае ограничения на значения $k_{1}$ не возникают.

Усечение разложения Хироты на данном уровне дает один диссипативный соли$\mathrm{TOH}$

$$
e^{( \pm)}= \pm 2 \beta \beta^{ \pm} e^{ \pm\left[k x+\left(k^{2}+2 \beta_{0}^{2}\right) \tau\right]} \frac{1+\gamma_{1}^{ \pm 1} e^{\tilde{\eta}_{1}}}{1+e^{\tilde{\eta}_{1}}},
$$

где мы включили константу $b_{1}$ в экспоненту: $\tilde{\eta}_{1}=k_{1} x+\omega_{1} \tau+\eta_{1}^{0}+\ln b_{1}$. В таком случае односолитонная плотность имеет вид

$$
\rho=-e^{(+)} e^{(-)}=\alpha_{1} \frac{\left(1+\gamma_{1} e^{\tilde{\eta}_{1}}\right)\left(1+\gamma_{1}^{-1} e^{\tilde{\eta}_{1}}\right)}{\left(1+e^{\tilde{\eta}_{1}}\right)^{2}} .
$$

Это решение может быть представлено в виде

$$
e^{( \pm)}= \pm \sqrt{\alpha_{1}} \mu^{ \pm 1} e^{ \pm\left[k x+\left(k^{2}+2 \beta_{0}^{2}\right) \tau\right]}\left(\frac{\gamma^{ \pm 1}+1}{2}+\frac{\gamma^{ \pm 1}-1}{2} \operatorname{th} \frac{\tilde{\eta}}{2}\right),
$$


или

$$
\begin{aligned}
& e^{(+)}=+\sqrt{\alpha_{1}} \frac{\mu}{2} e^{+\left[k x+\left(k^{2}+2 \beta_{0}^{2}\right) \tau\right]}\left(\gamma+1+(\gamma-1) \operatorname{th} \frac{\tilde{\eta}}{2}\right), \\
& e^{(-)}=-\sqrt{\alpha_{1}} \frac{1}{2 \mu} e^{-\left[k x+\left(k^{2}+2 \beta_{0}^{2}\right) \tau\right]}\left(\frac{1}{\gamma}+1+\left(\frac{1}{\gamma}-1\right) \operatorname{th} \frac{\tilde{\eta}}{2}\right),
\end{aligned}
$$

а произведение равно

$$
\rho=-e^{(+)} e^{(-)}=\alpha_{1}\left[1+\frac{(\gamma-1)^{2}}{4 \gamma \operatorname{ch}^{2}(\tilde{\eta} / 2)}\right]
$$

и имеет асимптотику $\lim _{|x| \rightarrow \infty} \rho \rightarrow \alpha_{1}$. В явном виде плотность равна

$$
\rho=-e^{(+)} e^{(-)}=\alpha_{1}\left\{1+\frac{k_{1}^{2}}{4 \beta_{0}^{2}} \operatorname{sch}^{2}\left[\frac{k_{1}}{2}\left(x+\left(2 k \pm \sqrt{k_{1}^{2}+4 \beta_{0}^{2}}\right) \tau+x_{0}\right)\right]\right\},
$$

где $\beta_{0}^{2}=1 /\left(4 \beta^{2} \alpha_{1}\right)$. Для поля скорости имеем

$$
u=\frac{e_{x}^{(-)}}{e^{(-)}}-\frac{e_{x}^{(+)}}{e^{(+)}}=-2 k-\frac{\left(\gamma^{2}-1\right) k_{1}}{(\gamma-1)^{2}+4 \gamma \operatorname{ch}^{2}(\tilde{\eta} / 2)} .
$$

Рассмотрим частное решение при $k=0$. Тогда соотношение

$$
\omega_{1}= \pm k_{1} \sqrt{k_{1}^{2}+4 \beta_{0}^{2}}
$$

есть дисперсионное соотношение Боголюбова в теории сверхтекучести слабо неидеального бозе-газа. При $k_{1} \gg 2 \beta_{0}$ оно имеет вид дисперсионного соотношения для свободной нерелятивистской частицы $\omega_{1} \approx k_{1}^{2}$, а при $k_{1} \ll 2 \beta_{0}$ - для релятивистского ансамбля $\omega_{1} \approx 2 \beta_{0} k_{1}$. Решение для положительного знака в формуле для дисперсии имеет вид

$$
\begin{aligned}
& e^{(+)}=\frac{\sqrt{\alpha_{1}} \mu}{v-\sqrt{v^{2}-4 \beta_{0}^{2}}} e^{2 \beta_{0}^{2} \tau}\left(v+\sqrt{v^{2}-4 \beta_{0}^{2}} \operatorname{th} \frac{\sqrt{v^{2}-4 \beta_{0}^{2}}}{2}\left(x+v \tau+x_{0}\right)\right), \\
& e^{(-)}=-\frac{\sqrt{\alpha_{1}} / \mu}{v+\sqrt{v^{2}-4 \beta_{0}^{2}}} e^{-2 \beta_{0}^{2} \tau}\left(v-\sqrt{v^{2}-4 \beta_{0}^{2}} \operatorname{th} \frac{\sqrt{v^{2}-4 \beta_{0}^{2}}}{2}\left(x+v \tau+x_{0}\right)\right),
\end{aligned}
$$

а плотность равна

$$
\rho=-e^{(+)} e^{(-)}=\alpha_{1}\left\{1+\frac{v^{2}-4 \beta_{0}^{2}}{4 \beta_{0}^{2}} \operatorname{sch}^{2}\left[\frac{\sqrt{v^{2}-4 \beta_{0}^{2}}}{2}\left(x+v \tau+x_{0}\right)\right]\right\} .
$$

Структура этого решения показывает, что скорость солитона ограничена снизу по модулю, $|v|>2 \beta_{0}$, так что солитон имеет "тахионный" характер. Полученные результаты означают, прежде всего, что в отличие от солитонов дефокусирующего НУШ, скорость которых ограничена сверху (дозвуковой тип), скорость солитонов РНУШ ограничена снизу (сверхзвуковой тип). Другое отличие состоит в том, что солитон дефокусирующего (отталкивающего) НУШ представляет собой возбуждение типа дырки (пузыря) с $|\psi|^{2}=\rho<1$, в то время как солитон РНУШ имеет форму типа барьера $\rho>1$. 
6.2.2. Двухсолитонное решение. Чтобы построить двухсолитонное решение, рассмотрим вслед за Хиротой [20] выражения

$$
g_{1}^{( \pm)}=a_{1}^{ \pm} e^{\eta_{1}}+a_{2}^{ \pm} e^{\eta_{2}}, \quad f_{1}=e^{\eta_{1}}+e^{\eta_{2}} .
$$

Подставляя их в билинейные уравнения

$$
\begin{gathered}
\left(\mp D_{\tau} \pm 2 k D_{x}+D_{x}^{2}\right)\left(a_{1}^{ \pm} e^{\eta_{1}}+a_{2}^{ \pm} e^{\eta_{2}}\right) \cdot 1+1 \cdot\left(e^{\eta_{1}}+e^{\eta_{2}}\right)=0, \\
2\left(D_{x}^{2}+2 \beta_{0}^{2}\right)\left(1 \cdot\left(e^{\eta_{1}}+e^{\eta_{2}}\right)\right)=2 \beta_{0}^{2}\left(a_{1}^{+} e^{\eta_{1}}+a_{2}^{+} e^{\eta_{2}}+a_{1}^{-} e^{\eta_{1}}+a_{2}^{-} e^{\eta_{2}}\right),
\end{gathered}
$$

имеем систему

$$
\begin{aligned}
& a_{1}^{ \pm}\left(\mp \partial_{\tau} \pm 2 k \partial_{x}+\partial_{x}^{2}\right) e^{\eta_{1}}+\left( \pm \partial_{\tau} \mp 2 k \partial_{x}+\partial_{x}^{2}\right) e^{\eta_{1}}+ \\
& \quad+a_{2}^{ \pm}\left(\mp \partial_{\tau} \pm 2 k \partial_{x}+\partial_{x}^{2}\right) e^{\eta_{2}}+\left( \pm \partial_{\tau} \mp 2 k \partial_{x}+\partial_{x}^{2}\right) e^{\eta_{2}}=0, \\
& \left(k_{1}^{2}+2 \beta_{0}^{2}\right)\left(e^{\eta_{1}}+e^{\eta_{2}}\right)=\beta_{0}^{2}\left[\left(a_{1}^{+}+a_{1}^{-}\right) e^{\eta_{1}}+\left(a_{2}^{+}+a_{2}^{-}\right) e^{\eta_{2}}\right] .
\end{aligned}
$$

Используя дисперсионные соотношения

$$
\omega_{i}^{ \pm}=k_{i}\left(2 k \pm \sqrt{k_{i}^{2}+4 \beta_{0}^{2}}\right), \quad i=1,2,
$$

получаем

$$
a_{i}^{+}=\frac{\left(\omega_{i}-2 k k_{i}\right)+k_{i}^{2}}{\left(\omega_{i}-2 k k_{i}\right)-k_{i}^{2}} \equiv e^{\phi_{i}}, \quad a_{i}^{-}=\frac{\left(\omega_{i}-2 k k_{i}\right)-k_{i}^{2}}{\left(\omega_{i}-2 k k_{i}\right)+k_{i}^{2}}=\frac{1}{a_{i}^{+}} e^{-\phi_{i}} .
$$

Последние соотношения означают, что

$$
\omega_{i}-2 k k_{i}=k_{i}^{2} \operatorname{cth} \frac{\phi_{i}}{2}
$$

и

$$
k_{i}=2 \beta_{0} \operatorname{sh} \frac{\phi_{i}}{2}
$$

так что

$$
\omega_{i}-2 k k_{i}=2 \beta_{0}^{2} \operatorname{sh} \phi_{i}
$$

Заметим, что два знака дисперсии в (71) отвечают простой замене $\phi_{i} \rightarrow-\phi_{i}$ в последних формулах. Ограничимся сначала рассмотрением одного и того же знака для обеих частот. В следующем порядке имеем систему

$$
\begin{gathered}
\left(\mp D_{\tau} \pm 2 k D_{x}+D_{x}^{2}\right)\left(g_{2}^{ \pm} \cdot 1+g_{1}^{ \pm} \cdot f_{1}+1 \cdot f_{2}\right)=0, \\
\left(D_{x}^{2}+2 \beta_{0}^{2}\right)\left(2 \cdot f_{2}+f_{1} \cdot f_{1}\right)=2 \beta_{0}^{2}\left(g_{2}^{+}+g_{2}^{-}+g_{1}^{+} g_{1}^{-}\right) .
\end{gathered}
$$

Первое уравнение переписывается как

$$
\begin{aligned}
\left(\mp \partial_{\tau}\right. & \left. \pm 2 k \partial_{x}+\partial_{x}^{2}\right) g_{2}^{ \pm}+\left( \pm \partial_{\tau} \mp 2 k \partial_{x}+\partial_{x}^{2}\right) f_{2}+ \\
& +\left\{a_{1}^{ \pm}\left[\mp\left(\omega_{1}-\omega_{2}\right) \pm 2 k\left(k_{1}-k_{2}\right)+\left(k_{1}-k_{2}\right)^{2}\right]+\right. \\
& \left.+a_{2}^{ \pm}\left[\mp\left(\omega_{2}-\omega_{1}\right) \pm 2 k\left(k_{2}-k_{1}\right)+\left(k_{1}-k_{2}\right)^{2}\right]\right\} e^{\eta_{1}+\eta_{2}}=0
\end{aligned}
$$


откуда следует, что оно имеет решение вида

$$
g_{2}^{ \pm}=a_{12}^{ \pm} e^{\eta_{1}+\eta_{2}}, \quad f_{2}=b_{12} e^{\eta_{1}+\eta_{2}} .
$$

Тогда из второго уравнения системы следуют соотношения

$$
a_{12}^{+}=a_{1}^{+} a_{2}^{+} b_{12}=e^{\phi_{1}+\phi_{2}} b_{12}, \quad a_{12}^{-}=a_{1}^{-} a_{2}^{-} b_{12}=e^{-\left(\phi_{1}+\phi_{2}\right)} b_{12},
$$

так что

$$
b_{12}=\frac{\operatorname{sh}^{2}\left(\left(\phi_{1}-\phi_{2}\right) / 4\right)}{\operatorname{sh}^{2}\left(\left(\phi_{1}+\phi_{2}\right) / 4\right)}
$$

при этом первое уравнение системы (76) выполняется автоматически. В итоге имеем решение

$$
e^{( \pm)}= \pm 2 \beta \frac{g_{0}^{ \pm}\left(1+g_{1}^{ \pm}+g_{2}^{ \pm}\right)}{1+f_{1}+f_{2}}
$$

или

$$
e^{( \pm)}= \pm 2 \beta \frac{g_{0}^{ \pm}\left(1+e^{\eta_{1} \pm \phi_{1}}+e^{\eta_{2} \pm \phi_{2}}+b_{12} e^{\eta_{1}+\eta_{2} \pm\left(\phi_{1}+\phi_{2}\right)}\right)}{1+e^{\eta_{1}}+e^{\eta_{2}}+b_{12} e^{\eta_{1}+\eta_{2}}} .
$$

В частном случае $\beta_{0}=1 / 2$ (при этом $\alpha_{1}=1$ ) имеем двухсолитонное решение для плотности $\rho$ :

$$
\rho=\frac{A_{+} A_{-}}{\left[\operatorname{sh}^{2}\left(\left(\phi_{1}+\phi_{2}\right) / 4\right)\left(1+e^{\eta_{1}}+e^{\eta_{2}}\right)+\operatorname{sh}^{2}\left(\left(\phi_{1}-\phi_{2}\right) / 4\right) e^{\eta_{1}+\eta_{2}}\right]^{2}},
$$

где

$$
\begin{aligned}
A_{ \pm} & =\operatorname{sh}^{2} \frac{\phi_{1}+\phi_{2}}{4}\left(1+e^{\eta_{1} \pm \phi_{1}}+e^{\eta_{2} \pm \phi_{2}}\right)+\operatorname{sh}^{2} \frac{\phi_{1}-\phi_{2}}{4} e^{\eta_{1}+\eta_{2} \pm\left(\phi_{1}+\phi_{2}\right)} \\
\eta_{i} & =\operatorname{sh} \frac{\phi_{i}}{2} x+\left[2 k \operatorname{sh} \frac{\phi_{i}}{2}+\frac{1}{2} \operatorname{sh} \phi_{i}\right] \tau+\eta_{i}^{(0)}, \quad i=1,2 .
\end{aligned}
$$

Если один из параметров $\phi_{i}$ обращается в нуль или если $\phi_{1}=\phi_{2}$, данное решение сводится к односолитонному. Например, если $\phi_{2}=0$, то

$$
\rho=1+\frac{\operatorname{sh}^{2}\left(\phi_{1} / 2\right)}{\operatorname{ch}^{2}\left(\eta_{1} / 2\right)}
$$

Исследуя двухсолитонное решение в движущихся системах отсчета, связанных с солитонами, можно видеть, что оно описывает столкновение двух солитонов типа (85), которые двигаются в одном направлении, имея начальные смещения

$$
\Delta x_{i}=(-1)^{i-1} \frac{2}{\operatorname{sh}\left(\phi_{i} / 2\right)} \ln \frac{\operatorname{sh}\left(\left(\phi_{1}-\phi_{2}\right) / 4\right)}{\operatorname{sh}\left(\left(\phi_{1}+\phi_{2}\right) / 4\right)}, \quad i=1,2,
$$

такие, что $\operatorname{sh}\left(\phi_{1} / 2\right) \Delta x_{1}+\operatorname{sh}\left(\phi_{2} / 2\right) \Delta x_{2}=0$.

Двухсолитонное решение другого вида получается, если выбрать противоположные знаки частот в (71), так что

$$
\begin{aligned}
& \omega_{1}^{+}=2 \beta_{0}\left(2 k \operatorname{sh} \frac{\phi_{1}}{2}+\beta_{0} \operatorname{sh} \phi_{1}\right), \\
& \omega_{2}^{-}=2 \beta_{0}\left(2 k \operatorname{sh} \frac{\phi_{1}}{2}-\beta_{0} \operatorname{sh} \phi_{1}\right) .
\end{aligned}
$$


Тогда $a_{1}^{ \pm}=e^{ \pm \phi_{1}}, a_{2}^{ \pm}=e^{\mp \phi_{2}}$,

$$
a_{12}^{+}=a_{1}^{+} a_{2}^{+} b_{12}=e^{\phi_{1}-\phi_{2}} b_{12}, \quad a_{12}^{-}=a_{1}^{-} a_{2}^{-} b_{12}=e^{-\phi_{1}+\phi_{2}} b_{12}
$$

и

$$
b_{12}=\frac{\operatorname{ch}^{2}\left(\left(\phi_{1}+\phi_{2}\right) / 4\right)}{\operatorname{ch}^{2}\left(\left(\phi_{1}-\phi_{2}\right) / 4\right)}
$$

При $\beta_{0}=1 / 2$ двухсолитонное решение имеет вид

$$
\rho=\frac{B_{+} B_{-}}{\left[\operatorname{ch}^{2}\left(\left(\phi_{1}-\phi_{2}\right) / 4\right)\left(1+e^{\eta_{1}}+e^{\eta_{2}}\right)+\operatorname{ch}^{2}\left(\left(\phi_{1}+\phi_{2}\right) / 4\right) e^{\eta_{1}+\eta_{2}}\right]^{2}},
$$

где

$$
\begin{aligned}
B_{ \pm} & =\operatorname{ch}^{2} \frac{\phi_{1}-\phi_{2}}{4}\left(1+e^{\eta_{1} \pm \phi_{1}}+e^{\eta_{2} \mp \phi_{2}}\right)+\operatorname{ch}^{2} \frac{\phi_{1}+\phi_{2}}{4} e^{\eta_{1}+\eta_{2} \pm \phi_{1} \mp \phi_{2}} \\
\eta_{1} & =\operatorname{sh} \frac{\phi_{1}}{2}\left(x-x_{1}\right)+\left[2 k \operatorname{sh} \frac{\phi_{1}}{2}+\frac{1}{2} \operatorname{sh} \phi_{1}\right] \tau \\
\eta_{2} & =\operatorname{sh} \frac{\phi_{2}}{2}\left(x-x_{2}\right)+\left[2 k \operatorname{sh} \frac{\phi_{2}}{2}-\frac{1}{2} \operatorname{sh} \phi_{2}\right] \tau .
\end{aligned}
$$

Это решение описывает столкновение двух солитонов вида (85), которые двигаются в противоположных направлениях, имея начальные смещения

$$
\Delta x_{i}=(-1)^{i-1} \frac{2}{\operatorname{sh}\left(\phi_{i} / 2\right)} \ln \frac{\operatorname{ch}\left(\left(\phi_{1}+\phi_{2}\right) / 4\right)}{\operatorname{ch}\left(\left(\phi_{1}-\phi_{2}\right) / 4\right)}, \quad i=1,2 .
$$

На рисунке представлен трехмерный график такого решения.

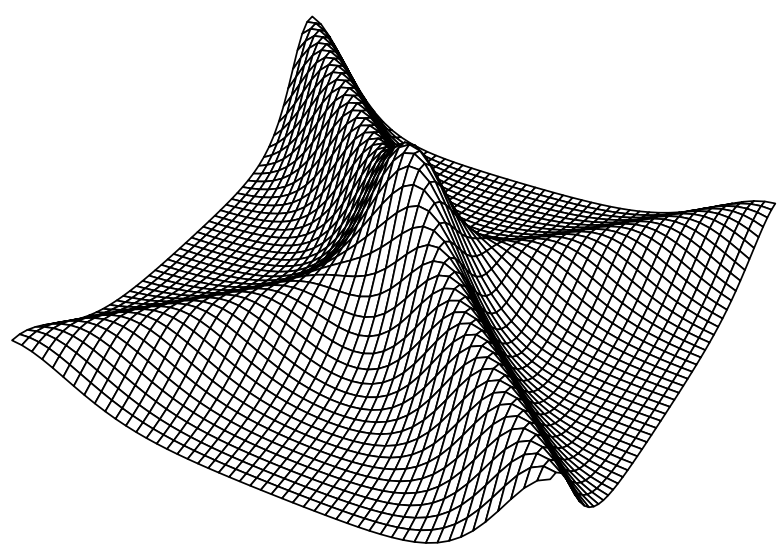


Для поля скоростей имеем

$$
\begin{aligned}
& u=\frac{e_{x}^{(-)}}{e^{(-)}}-\frac{e_{x}^{(+)}}{e^{(+)}}=-2 k+ \\
& \quad+\frac{\operatorname{ch}^{2}\left(\left(\phi_{1}-\phi_{2}\right) / 4\right) a_{-}+\operatorname{ch}^{2}\left(\left(\phi_{1}-\phi_{2}\right) / 4\right) b_{-}}{\operatorname{ch}^{2}\left(\left(\phi_{1}-\phi_{2}\right) / 4\right)\left(1+e^{\eta_{1}-\phi_{1}}+e^{\eta_{2}+\phi_{2}}\right)+\operatorname{ch}^{2}\left(\left(\phi_{1}+\phi_{2}\right) / 4\right) e^{\eta_{1}+\eta_{2}-\phi_{1}+\phi_{2}}}- \\
& \quad-\frac{\operatorname{ch}^{2}\left(\left(\phi_{1}-\phi_{2}\right) / 4\right) a_{+}+\operatorname{ch}^{2}\left(\left(\phi_{1}-\phi_{2}\right) / 4\right) b_{+}}{\operatorname{ch}^{2}\left(\left(\phi_{1}-\phi_{2}\right) / 4\right)\left(1+e^{\eta_{1}+\phi_{1}}+e^{\eta_{2}-\phi_{2}}\right)+\operatorname{ch}^{2}\left(\left(\phi_{1}+\phi_{2}\right) / 4\right) e^{\eta_{1}+\eta_{2}+\phi_{1}-\phi_{2}}},
\end{aligned}
$$

где

$$
a_{\mp}=\operatorname{sh} \frac{\phi_{1}}{2} e^{\eta_{1} \mp \phi_{1}}+\operatorname{sh} \frac{\phi_{2}}{2} e^{\eta_{2} \pm \phi_{2}}, \quad b_{\mp}=\left(\operatorname{sh} \frac{\phi_{1}}{2}+\operatorname{sh} \frac{\phi_{2}}{2}\right) e^{\eta_{1}+\eta_{2} \mp \phi_{1} \pm \phi_{2}} .
$$

Это решение имеет такой же сдвиг фаз (92) и описывает столкновение двух солитонов вида

$$
u=-2 k-\frac{k_{i} \operatorname{sh} \phi_{i}}{2 \operatorname{ch}\left(\left(\eta_{i}+\phi_{i}\right) / 2\right) \operatorname{ch}\left(\left(\eta_{i}-\phi_{i}\right) / 2\right)}, \quad i=1,2 .
$$

Благодарности. Эта работа была частично поддержана Institute of Mathematics, Academia Sinica (Taipei, Taiwan) и Izmir Institute of Technology (Izmir, Turkey), проект BAP 2005 IYTE 08.

\section{Список литературы}

[1] O. K. Pashaev, J.-H. Lee, Mod. Phys. Lett. A, 17 (2002), 1601-1619.

[2] L. de Broglie, C. R. Acad. Sci. Paris, 183 (1926), 447-448.

[3] D. Bohm, Phys. Rev., 85 (1952), 166-179; 180-193.

[4] E. Nelson, Phys. Rev., 150 (1966), 1079-1085.

[5] G. Salesi, Mod. Phys. Lett. A, 11 (1996), 1815-1823.

[6] F. Guerra, M. Pusterla, Lett. Nuovo Cimento (2), 34 (1982), 351-356.

[7] L. Smolin, Phys. Lett. A, 113:8 (1986), 408-412.

[8] O. Bertolami, Phys. Lett. A, 154 (1991), 225-229.

[9] L. K. Antanovskii, C. Rogers, W. K. Schief, J. Phys. A, 30 (1997), L555-L557.

[10] C. Rogers, W. K. Schief, Nuovo Cimento B, 114 (1999), 1409-1412.

[11] J.-H. Lee, O. K. Pashaev, C. Rogers, W. K. Schief, J. Plasma Phys., 73:02 (2007), 257-272.

[12] В.И. Карпман, Нелинейные волны в диспергирующих средах, Наука, М., 1973.

[13] А. И. Ахиезер, И. А. Ахиезер, Р. В. Половин, А. Г. Ситенко, К. Н. Степанов, Электродинамика плазмы, Наука, М., 1974.

[14] J. H. Adlam, J. E. Allen, Philos. Mag., 3 (1958), 448-455.

[15] А. В. Гуревич, А. П. Мещеркин, ЖЭТФ, 87 (1984), 1277-1292.

[16] G. A. El, V. V. Khodorovskii, A. V. Tyurina, Phys. Lett. A, 333 (2004), 334-340.

[17] А. В. Гуревич, А. Л. Крылов, ЖЭЭТФ, 92 (1987), 1684-1699.

[18] А. В. Гуревич, А. Л. Крылов, Докл. АН СССР, 298 (1988), 608-611.

[19] G. A. El, R. H. J. Grimshaw, M. V. Pavlov, Stud. Appl. Math., 106 (2001), 157-186.

[20] R. Hirota, "Direct method of finding exact solutions of nonlinear evolution equations", Bäcklund Transformations, the Inverse Scattering Method, Solitons, and Their Applications (Nashville, 1974), Lecture Notes in Math., 515, ed. R. M. Miura, Springer, Berlin, 1976, $40-68$. 